\title{
Ellipsometric Raman Spectroscopy
}

Fernando C. Basilio ${ }^{\S}$, Patricia T. Campana ${ }^{*}$, Eralci M. Therézio ${ }^{*}$, Newton M. Barbosa Neto ${ }^{\#}$, Françoise Serein-Spirau ${ }^{\S \S}$, Raigna A. Silva ${ }^{\S}$, Osvaldo N. Oliveira Jr. ${ }^{\circledR}$, Alexandre Marletta ${ }^{\S}$

${ }^{\S}$ Physics Institute, Federal University of Uberlândia, 38400-902, Uberlândia - MG, Brazil

${ }^{\dagger}$ School of Art, Sciences and Humanities, University of São Paulo, 03828-000, São Paulo-SP, Brazil.

$\$$ Mathematic Department, Federal University of Mato Grosso, 78735-901, Rondonópolis - MT, Brazil.

\#Institute of Exact and Natural Sciences, Federal University of Pará, 66075-110, Belém - PA, Brazil.

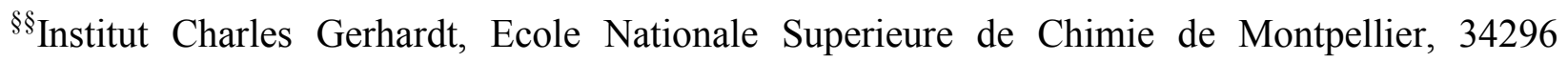
Montpellier, France.

\&Physics Institute of São Carlos, University of São Paulo, CP 369, 13560-970, São Carlos-SP, Brazil.

Corresponding Author: *Patricia T. Campana. Phone number: +55 1130918883. Mobile/whatsapp: +55 11 982410290. E-mail: pcampana@usp.br 


\section{Degree of polarization - $P$}

The degree of polarization of the analyzed light is defined as:

$\mathrm{P}=\frac{\mathrm{I}_{\mathrm{pol}}}{\mathrm{I}_{\mathrm{tot}}}=\frac{\left(\mathrm{S}_{1}^{2}+\mathrm{S}_{2}^{2}+\mathrm{S}_{3}^{2}\right)^{1 / 2}}{\mathrm{~S}_{0}}$

where $I_{p o l}$ is the intensity of the polarized components and $I_{\text {tot }}$ is the total intensity of the beam. $\mathrm{P}=1$ corresponds to fully polarized light, $\mathrm{P}=0$ when light is non-polarized and $0<$ $P<1$ corresponds to partially polarized light. The degree of polarization $\mathrm{P}$ does not indicate whether polarization is linear or circular; it only provides information on how much of the light is polarized.

\section{Anisotropy - r}

The anisotropy factor, $r$, is calculated as:

$\mathrm{r}=\frac{\mathrm{I}_{\mathrm{N}, \mathrm{ll}}-\mathrm{GI}_{\mathrm{I}, \perp}}{\mathrm{I}_{\mathrm{I}, \mathrm{I}}+2 \mathrm{GI} \mathrm{I}_{\|, \perp}}$

where $I_{\|,\|}$is the scattered light due to excitation with parallel polarization and detected with the polarizer at the parallel direction; $I_{\|, \perp}$ is the scattered light owing to excitation with parallel polarization and detected with the polarizer in the perpendicular direction. The laboratory reference frame was adopted, in which the vertical direction is parallel and the horizontal direction is perpendicular. The factor $G$ is defined as:

$\mathrm{G}=\frac{\mathrm{I}_{\perp, \|}}{\mathrm{I}_{\mathrm{I}, \mathrm{I}}}$

where $I_{\perp, \|}$ is the scattered light in perpendicular direction and excited at parallel direction and $I_{\|,\|}$is the scattered and detected light in parallel direction. $G$ is used to correct the effects from optical components on the polarization degree of the analyzed scattered light. Since for ERS the analyzer is fixed, there is no need to make this correction, which is advantageous because the 
number of measurements required is less and the Stokes parameters may be obtained directly. For an impinging linearly polarized light, the intensity at the sample will be $S_{0}$ and the difference in intensity between the vertical and horizontal polarized lights will be given by $S_{1}{ }^{\mathrm{S} 1}$. With some algebraic manipulation, it can be shown that:

$r_{E E}=-2 \frac{s_{1} / s_{0}}{{ }^{3+{ }} / s_{0}}$.

In contrast to the conventional expression in eq. S2, where $r$ varies from -1 to $1, r_{E E}$ varies between $-1 / 2$ and 1 .

\section{Asymmetry - g}

The asymmetry factor $\mathrm{g}$ is defined as:

$\mathrm{g}=-2 \frac{\mathrm{I}^{\mathrm{R}}-\mathrm{I}^{\mathrm{L}}}{\mathrm{I}^{\mathrm{R}}+\mathrm{I}^{\mathrm{L}}}$

where $I^{R}$ and $I^{L}$ are the scattered light intensities with excitation circularly polarized at the right (R) and at the left (L). It is related to the parameter $\Delta$, which is the difference in circularly polarized scattered light, with $\Delta=-\frac{g}{\underline{2}}$. In the new formulation presented here, $g$ is obtained from the Stokes parameters (eq. 2), from the scattered light with linearly polarized excitation. Since we may decompose a linearly polarized light as the sum of two circularly polarized lights (at the right and left), the Stokes parameter S3 gives the difference $I^{R}-I^{L}$, while S0 gives the sum of intensities $I^{R}+I^{L}$ in Eq. 1. Hence,g is given by ${ }^{\mathrm{S} 2}$ :

$g=2 \frac{S_{3}}{S_{0}}$.

Angle $\psi$ and ellipticity $\chi$ 
Consider a rotated ellipsis as the most general case of polarization. The elliptically polarized light may be related to the Stokes parameters via the geometrical parameters in the form:

$$
\begin{aligned}
& \tan (2 \psi)=\frac{s_{2}}{s_{1}} \\
& \tan (2 \chi)=\frac{s_{3}}{s_{1}}
\end{aligned}
$$

where $\psi$ is the orientation angle and $\chi$ is the ellipticity of an elliptically polarized light with axes $a$ and $b$.

\section{Mueller Matrix for a polarizer}

An incident beam with electric field components $E_{x}$ and $E_{y}$ is made to pass through a polarizer, the components of the emerging field being $E_{x}^{\prime}$ and $E_{y}^{\prime}$ :

$E_{x}^{\prime}=p_{x} E_{x}$

$E_{y}^{\prime}=p_{y} E_{y}$

where $p_{x}$ and $p_{y}$ are the attenuation coefficients for each of the axes of the impinging beam, with $0 \leq p_{x}\left(p_{y}\right) \leq 1$. For an ideal polarizer, there is only transmission $\left(p_{x}\right.$ or $\left.p_{y}=1\right)$ of the component in one direction, while the other component is entirely absorbed $\left(p_{x}\right.$ or $\left.p_{y}=0\right)$. In the general case:

$$
M_{P}=\frac{1}{2}\left(\begin{array}{cccc}
p_{x}^{2}+p_{y}^{2} p_{x}^{2}-p_{y}^{2} & 0 & 0 \\
p_{x}^{2}-p_{y}^{2} p_{x}^{2}+p_{y}^{2} & 0 & 0 \\
0 & 0 & 2 p_{x} p_{y} & 0 \\
0 & 0 & 0 & 2 p_{x} p_{y}
\end{array}\right)
$$

For an ideal polarizer with vertical polarization (in the laboratory reference frame), $p_{y}=1$ and $p_{x}=0$, thus yielding: 
$M_{P}=\frac{1}{2}\left(\begin{array}{rrrr}1 & -1 & 0 & 0 \\ -1 & 1 & 0 & 0 \\ 0 & 0 & 0 & 0 \\ 0 & 0 & 0 & 0\end{array}\right)$

\section{Mueller matrix for a rotator}

The Mueller matrix is altered when an optical component is rotated by $\theta$ in relation to $\mathrm{x}$ or y. Using a rotor depicted in Figure S1, where $\theta$ is the angle between the rotated axes in relation to the system with coordinates $E_{x}$ and $E_{y}$, and $\beta$ is the angle between $E$ and $E_{x}$, the coordinates for the rotated axes are given by:

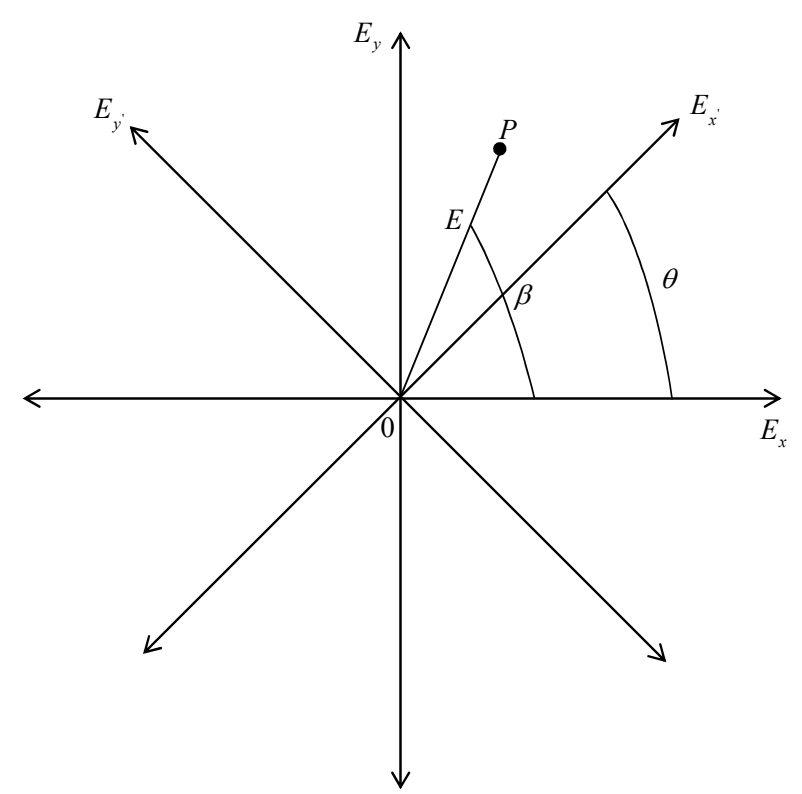

Figure S1. Rotation of an electric field by a rotor $^{\mathrm{S} 3}$.

$E_{x}^{\prime}=E_{x} \cos \theta+E_{y} \sin \theta$

$E_{y}^{\prime}=-E_{x} \sin \theta+E_{y} \cos \theta$

The resulting Mueller matrix is: 
$M_{R}(2 \theta)=\left(\begin{array}{cccc}1 & 0 & 0 & 0 \\ 0 & \cos (2 \theta) & \sin (2 \theta) & 0 \\ 0 & -\sin (2 \theta) & \cos (2 \theta) & 0 \\ 0 & 0 & 0 & 1\end{array}\right)$

In order to obtain the Mueller matrix for a rotated component, one considers the impinging beam on a rotor. For a quarter-wave retarder rotated by $\theta$ with regard to the original axes:

$$
M_{\lambda / 4}(2 \theta)=\left(\begin{array}{cccc}
1 & 0 & 0 & 0 \\
0 & \cos ^{2}(2 \theta) & \operatorname{sen}(2 \theta) \cos (2 \theta) & -\operatorname{sen}(2 \theta) \\
0 \operatorname{sen}(2 \theta) \cos (2 \theta) & \operatorname{sen}^{2}(2 \theta) & \cos (2 \theta) \\
0 & \sin (2 \theta) & -\cos (2 \theta) & 0
\end{array}\right)
$$

\section{Forward $\left(0^{\circ}\right)$ and backward $\left(180^{\circ}\right)$ scattering}

a) Stokes parameters for forward scattering:

$S_{0}^{d}\left(0^{o}\right)=\frac{1}{2} K\left\{6 \tilde{\alpha}_{\alpha \beta} \tilde{\alpha}_{\alpha \beta}^{*}+2 \tilde{\alpha}_{\alpha \beta} \tilde{\alpha}_{\beta \alpha}^{*}+\tilde{\alpha}_{\alpha \alpha} \tilde{\alpha}_{\beta \beta}^{*}-\frac{2}{c} \operatorname{Im}\left[\frac{1}{3} i \omega \epsilon_{\alpha \gamma \delta}\left(\tilde{\alpha}_{\alpha \beta} \tilde{\mathcal{A}}_{\gamma \delta \beta}^{*}+2 \tilde{\alpha}_{\beta \alpha} \tilde{\mathcal{A}}_{\gamma \delta \beta}^{*}-\right.\right.\right.$ $\left.\left.\left.\tilde{\alpha}_{\beta \alpha} \tilde{A}_{\gamma \delta \beta}^{*}\right)\right]\right\}$

$S_{1}^{d}\left(0^{o}\right)=K\left\{-2 \tilde{\alpha}_{\alpha \beta} \tilde{\alpha}_{\alpha \beta}^{*}+3 \tilde{\alpha}_{\alpha \beta} \tilde{\alpha}_{\beta \alpha}^{*}+3 \tilde{\alpha}_{\alpha \alpha} \tilde{\alpha}_{\beta \beta}^{*}\right\}$

$S_{2}^{d}\left(0^{o}\right)=K \frac{2}{c} \operatorname{Re}\left[\frac{1}{3} i \omega \epsilon_{\alpha \gamma \delta}\left(-\tilde{\alpha}_{\beta \alpha} \tilde{A}_{\gamma \delta \beta}^{*}-\tilde{\alpha}_{\beta \alpha} \tilde{A}_{\gamma \delta \beta}^{*}\right)-2 \tilde{\alpha}_{\alpha \beta} \tilde{G}_{\alpha \beta}^{*}+3 \tilde{\alpha}_{\alpha \beta} \tilde{G}_{\beta \alpha}^{*}+3 \tilde{\alpha}_{\alpha \alpha} \tilde{G}_{\beta \beta}^{*}+\right.$ $\left.2 \tilde{\alpha}_{\alpha \beta} \widetilde{\Xi}_{\alpha \beta}^{*}-3 \tilde{\alpha}_{\alpha \beta} \widetilde{\Xi}_{\beta \alpha}^{*}-3 \widetilde{\Xi}_{\beta \beta}^{*}\right]$

$S_{3}^{d}\left(0^{o}\right)=K \frac{2}{c} \operatorname{Im}\left[\frac{1}{3} i \omega \epsilon_{\alpha \gamma \delta}\left(-2 \tilde{\alpha}_{\alpha \beta} \tilde{A}_{\gamma \delta \beta}-\tilde{\alpha}_{\beta \alpha} \tilde{A}_{\gamma \delta \beta}^{*}+\tilde{\alpha}_{\alpha \beta} \tilde{\mathcal{A}}_{\gamma \delta \beta}\right)+5 \tilde{\alpha}_{\alpha \alpha} \tilde{G}_{\beta \beta}^{*}-5 \tilde{\alpha}_{\alpha \beta} \tilde{G}_{\beta \alpha}^{*}-\right.$ $\left.6 \tilde{\alpha}_{\alpha \beta} \widetilde{\Xi}_{\alpha \beta}^{*}-\tilde{\alpha}_{\alpha \beta} \widetilde{\Xi}_{\beta \alpha}^{*}-3 \tilde{\alpha}_{\alpha \alpha} \widetilde{\Xi}_{\beta \beta}^{*}\right]$

\section{b) Stokes parameters for backward scattering}

$S_{0}^{d}\left(180^{\circ}\right)=K\left\{6 \tilde{\alpha}_{\alpha \beta} \tilde{\alpha}_{\alpha \beta}^{*}+\tilde{\alpha}_{\alpha \beta} \tilde{\alpha}_{\beta \alpha}^{*}+\tilde{\alpha}_{\alpha \alpha} \tilde{\alpha}_{\beta \beta}^{*}\right\}$

$S_{1}^{d}\left(180^{\circ}\right)=K\left\{-2 \tilde{\alpha}_{\alpha \beta} \tilde{\alpha}_{\alpha \beta}^{*}+3 \tilde{\alpha}_{\alpha \beta} \tilde{\alpha}_{\beta \alpha}^{*}+3 \tilde{\alpha}_{\alpha \alpha} \tilde{\alpha}_{\beta \beta}^{*}\right\}$

$S_{2}^{d}\left(180^{\circ}\right)=\frac{K}{c} \operatorname{Re}\left[\frac{2}{3} i \omega \epsilon_{\alpha \gamma \delta}\left(-\tilde{\alpha}_{\beta \alpha} \tilde{A}_{\gamma \delta \beta}+\tilde{\alpha}_{\alpha \beta} \tilde{\mathcal{A}}_{\gamma \delta \beta}\right)-2 \tilde{\alpha}_{\alpha \beta} \tilde{G}_{\alpha \beta}^{*}+3 \tilde{\alpha}_{\alpha \beta} \tilde{G}_{\beta \alpha}^{*}+3 \tilde{\alpha}_{\alpha \alpha} \tilde{G}_{\beta \beta}^{*}-\right.$ $\left.2 \tilde{\alpha}_{\alpha \beta} \widetilde{\Xi}_{\alpha \beta}^{*}+3 \tilde{\alpha}_{\alpha \beta} \widetilde{\Xi}_{\beta \alpha}^{*}+3 \tilde{\alpha}_{\alpha \alpha} \widetilde{\Xi}_{\beta \beta}^{*}\right]$ 


$$
\begin{aligned}
& S_{3}^{d}\left(180^{\circ}\right)=K \frac{2}{c} \operatorname{Im}\left[-\frac{2}{3} i \omega \epsilon_{\alpha \gamma \delta}\left(-2 \tilde{\alpha}_{\alpha \beta} \tilde{A}_{\gamma \delta \beta}-\tilde{\alpha}_{\beta \alpha} \tilde{A}_{\gamma \delta \beta}^{*}+\tilde{\alpha}_{\alpha \beta} \tilde{\mathcal{A}}_{\gamma \delta \beta}\right)-5 \tilde{\alpha}_{\alpha \beta} \tilde{G}_{\beta \alpha}^{*}+\right. \\
& \left.5 \tilde{\alpha}_{\alpha \alpha} \tilde{G}_{\beta \beta}^{*}+6 \tilde{\alpha}_{\alpha \beta} \widetilde{\Xi}_{\alpha \beta}^{*}+\tilde{\alpha}_{\alpha \beta} \widetilde{\Xi}_{\beta \alpha}^{*}+\tilde{\alpha}_{\alpha \alpha} \widetilde{\Xi}_{\beta \beta}^{*}\right]
\end{aligned}
$$

$$
\text { where } K=\frac{1}{30}\left(\omega^{2} \mu_{o} E^{(0)} / 4 \pi R\right)^{2} \text {. }
$$

\section{Testing the 9-point method}

The efficiency of the 9-point method was tested with the analysis of a vertical linearly polarized light of an argon laser at $514.6 \mathrm{~nm}$ and of a non-polarized He-Ne laser at $633 \mathrm{~nm}$. The measurements were performed in $40^{\circ}$ steps with the apparatus in Figure 1 (main text) by removing the Notch (F2) filter and replacing the argon laser by the He-Ne laser. Figure S2a shows the intensity $I(\theta, \lambda)$ (Eq. 2), where there is a significant difference for the polarized 514.6 $\mathrm{nm}$ light, as expected, but no difference for the $633 \mathrm{~nm}$, which is non-polarized. The Stokes parameters, normalized with respect to the total intensity $\left(S_{0}\right)$ without considering a baseline $S_{0}^{B L}=0$ and with baseline $S_{0}^{B L} \ll S_{0}^{E R S} \neq 0$, are shown in Figures $\mathrm{S} 2 \mathrm{~b}$ and SI2c, respectively. These conditions also apply to $P$ (eq. S2), $r_{E E}$ (eq. S4) and $g$ (eq. S6) in Figures S2d and S2e. When $S_{0}=0$, the Stokes parameters are undefined owing to a 0/0 ratio (see Figures S2b and S2d), as mentioned before for $\Delta$ (Eq. 1, main text). However, adding a baseline does not affect the parameters significantly, provided that $I_{0}^{B L}$ is much smaller than $I(\theta, \lambda)$. This allows for the inconsistency in the regions where $S_{0}=0$ to be removed, as seen in Figures S2c and S2e.This methodology was also adopted for the Raman spectra. 

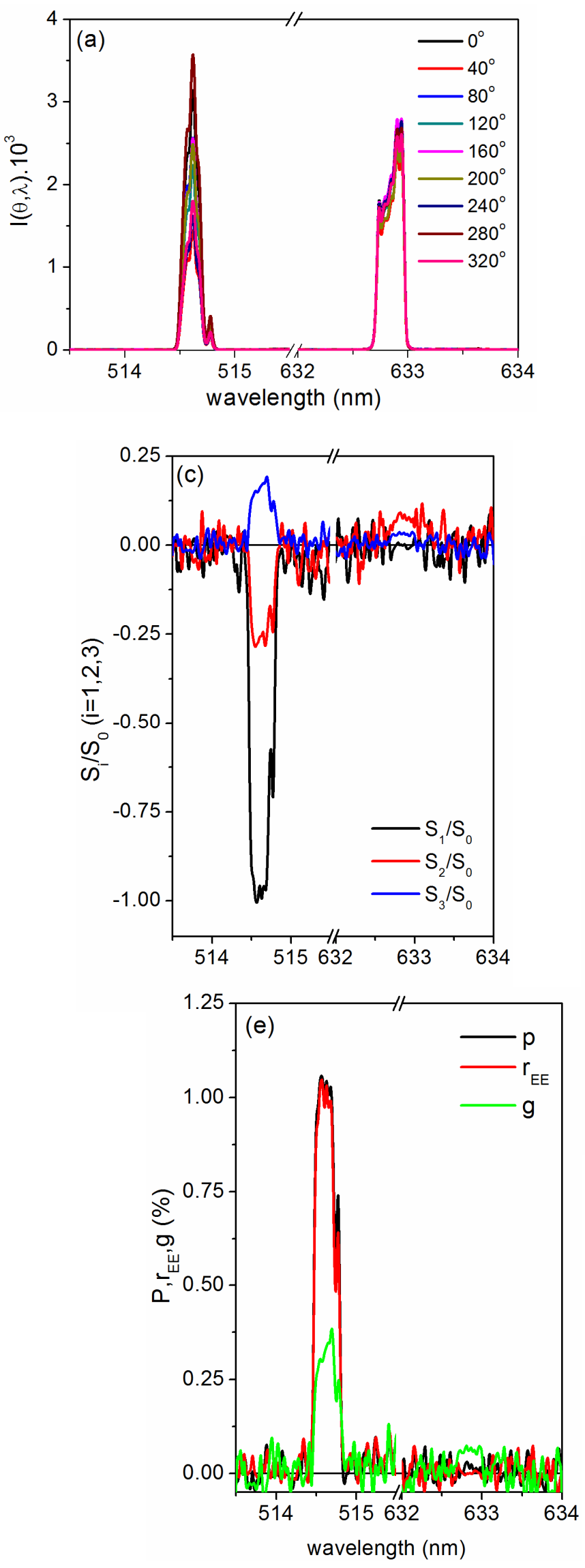
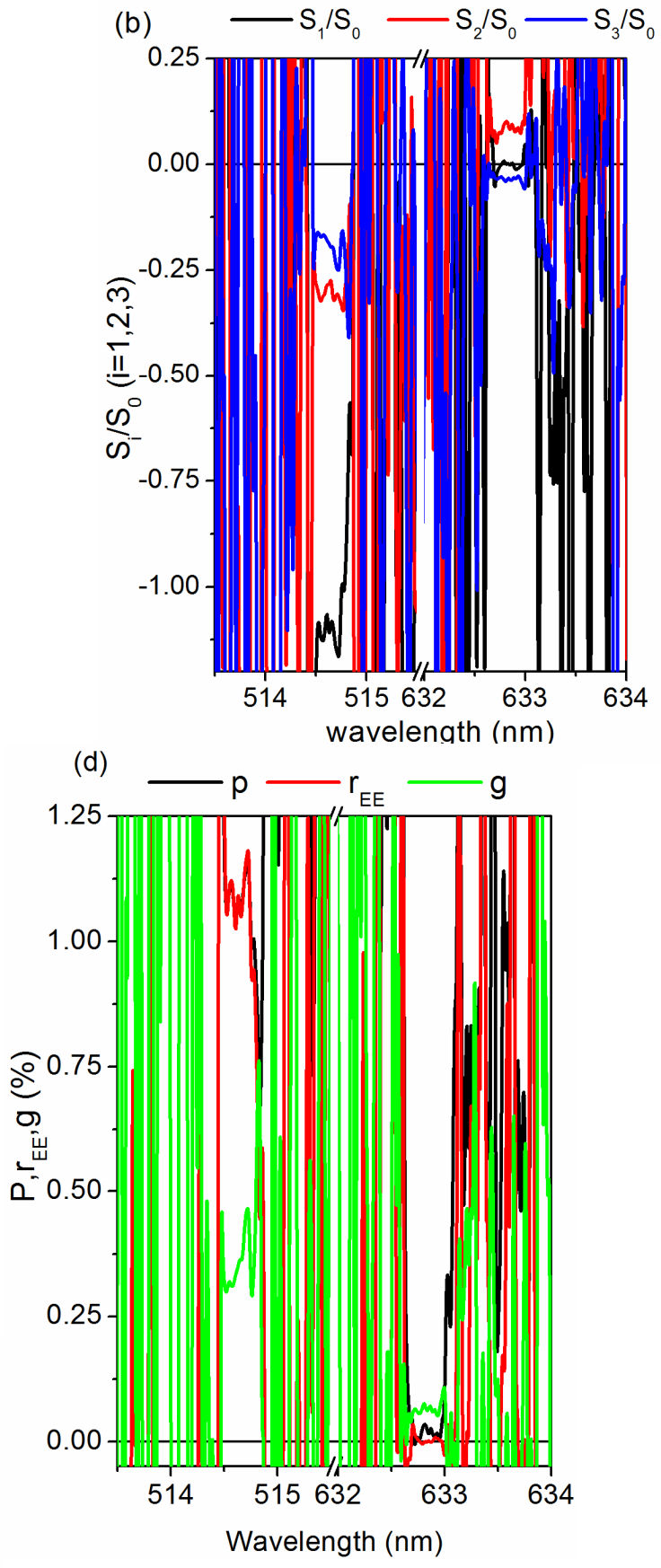
Figure S2.Normalized Stokes Parameters $\left(S_{i}^{d} / S_{\mathbf{0}}^{\boldsymbol{d}} ; \boldsymbol{i}=\mathbf{1}, \mathbf{2}, \mathbf{3}\right)$ for emission ellipsometry with linearly polarized light in the vertical.

From Table $\mathrm{S} 1$, one notes that for the linearly polarized light in the vertical $(514.6 \mathrm{~nm}), S_{1} / S_{0}$ is large and negative, which means that most of the impinging light is linearly polarized in the vertical. A smaller portion is linearly polarized at $+45^{\circ}$, as seen with parameters $S_{2} / S_{0}$ and $S_{3} / S_{0}$. These results show that the analyzed light is not $100 \%$ polarized as intended, owing to the difficulty in aligning the optical system (mirrors, polarizers, quarter-wave plate). For the 633nm, which should be fully non-polarized, there is a small degree of polarization, again owing to the difficulty in alignment. Nevertheless, these results confirm the efficiency of the methodology in which only 9 points were used, thus minimizing the number of required measurements. This is crucial for samples that photodegrade rapidly, for example.

Table S1. Normalized Stokes parameters and parameters $P$ (eq. S1), $r_{E E}$ (eq. S4) and $g$ (eq. S6) for emission ellipsometry with $514.6 \mathrm{~nm}$ and $633 \mathrm{~nm}$ lasers, for the vertical linearly polarized (PLV) and non-polarized (DP) lights.

\begin{tabular}{llllllll} 
Polarization & $\lambda(\mathrm{nm})$ & $\mathrm{S}_{1} / \mathrm{S}_{0}$ & $\mathrm{~S}_{2} / \mathrm{S}_{0}$ & $\mathrm{~S}_{3} / \mathrm{S}_{0}$ & $P$ & $r_{E E}$ & $g$ \\
& & $( \pm 0.02)$ & $( \pm 0.02)$ & $( \pm 0.02)$ & $( \pm 0.02)$ & $( \pm 0.02)$ & $( \pm 0.02)$ \\
\hline PLV & 514.6 & -0.96 & 0.22 & 0.02 & 1.02 & 0.52 & 0.35 \\
\hline DP & 633.0 & +0.05 & -0.07 & 0.02 & 0.04 & -0.04 & 0.07 \\
\hline
\end{tabular}

Another worth noting issue is the symmetry of the ellipsometric curve. Measurements with two angles differing by $180^{\circ}$ must be performed and verify whether the results are identical, as they should because the emission ellipsometry curve has a periodicity of $180^{\circ}$. Furthermore, the 
Stokes parameters may be calculated for each wavelength, owing to the achromatic quarter-wave plate and polarizer. This allows one to obtain the emission and/or scattering spectrum for the sample via the parameter S0, regardless of the geometry and optical components used.

\section{SW254 possible configurations}

Proposed configurations for SW254 monomers and for the dimeric molecules $(n=2)$, generated by $\mathrm{ACD} /$ ChemSketch Advanced Chemistry Development, Inc. ${ }^{\mathrm{S}}$

a)

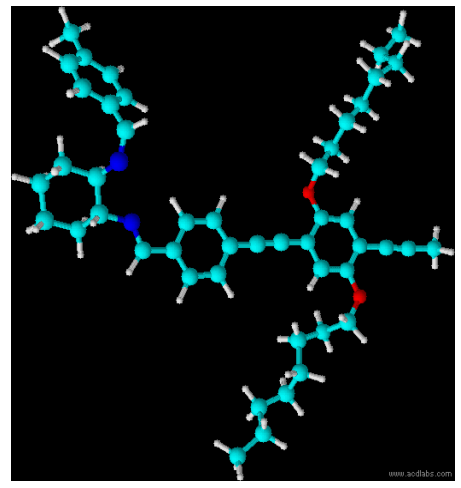

b)
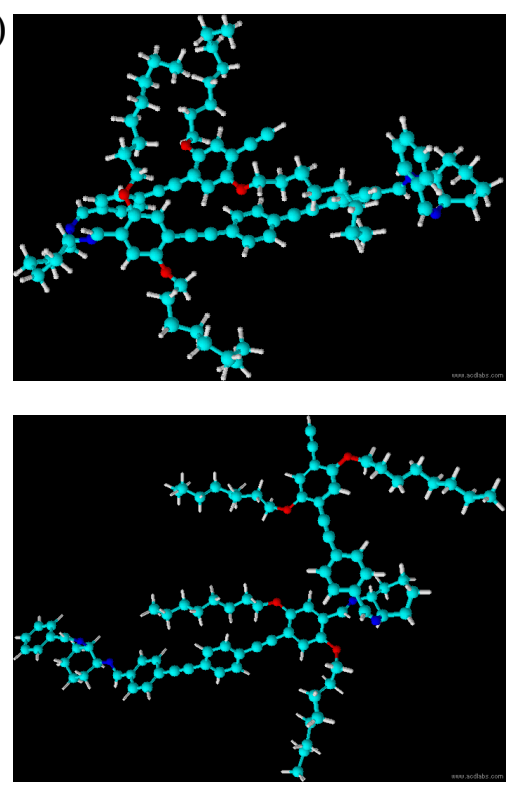
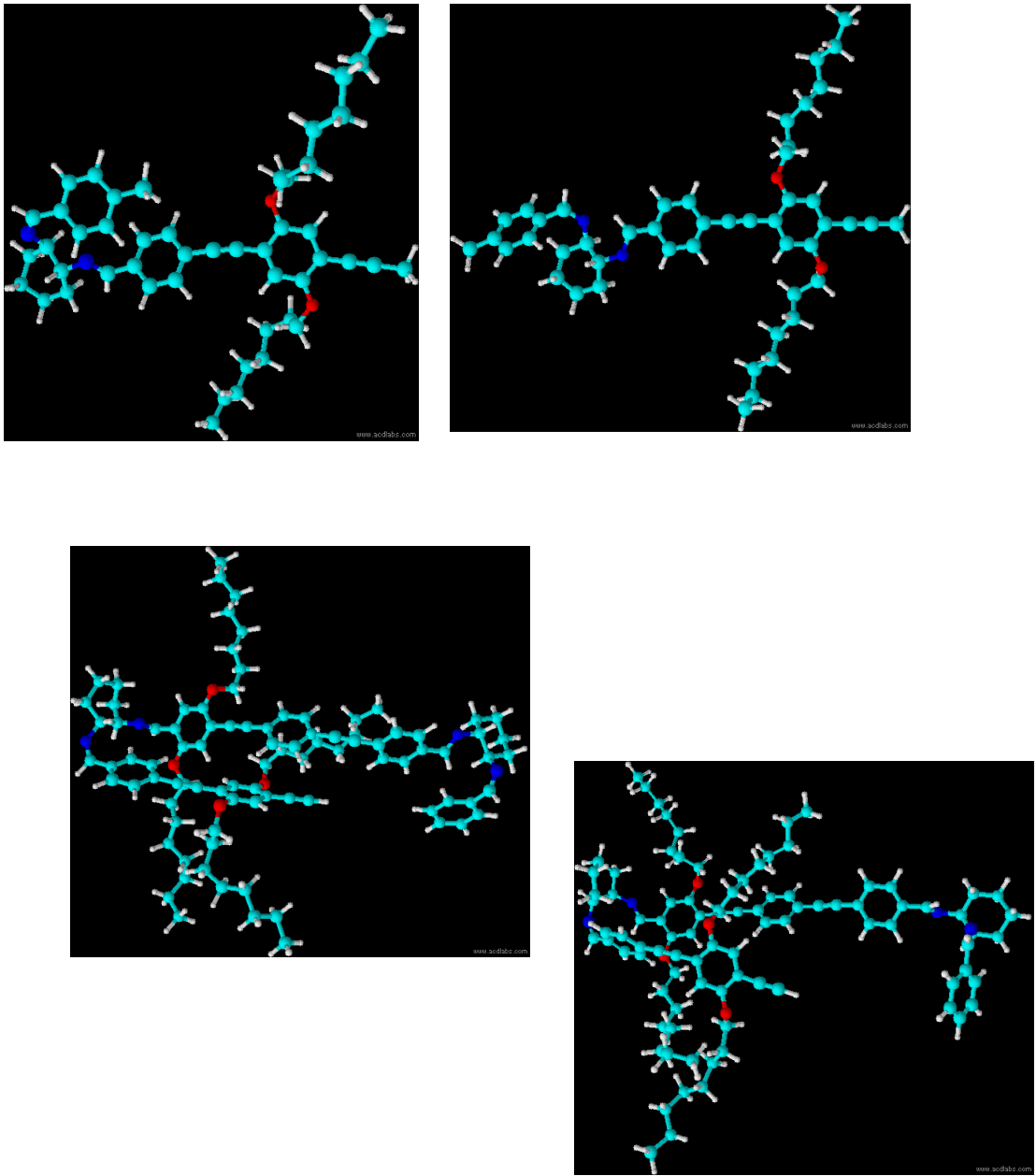

Figure S3.Proposed conformations for SW254 monomers (a) and for the dimeric molecules $(n=2)(b)$. 


\section{REFERENCES}

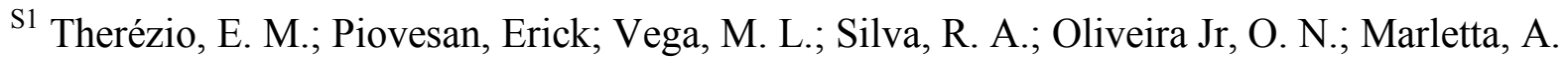
Thickness and Annealing Temperature Effects on the Optical Properties and Surface Morphology of Layer-by-Layer Poly(p-phenyline vinylene) plus Dodecylbenzenesulfonate Films. J. Polym. Sci., Part B: Polym. Phys. 2011, 49, 206-213. DOI: 10.1002/polb.22180

S2 Alliprandini Filho. P. Aplicação da Técnica de Elipsometria de Emissão para Caracterização de Materiais Luminescentes. Ph.D. Thesis, Federal university of Uberlândia, March 2012.

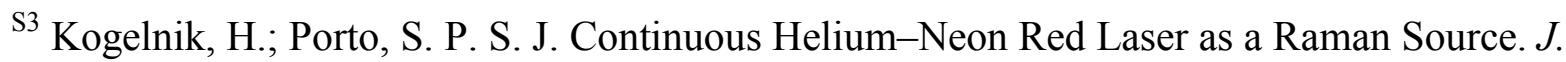
Opt. Soc. Am. 1963, 53(12), 1446-1447.

${ }^{\mathrm{S} 4} \mathrm{ACD} / \mathrm{Chem}$ Sketch Freeware, version 12.01, Advanced Chemistry Development, Inc., Toronto, On, Canada, 2015. Home Page. www.acdlabs.com (accessed Jul 5, 2016). 